Background Primary hepatocellular carcinoma (HCC) is one of the most common malignancies all over the world. However, the mechanism of HCC initiation and development remains unclear. In our previous work, high-throughput microarray assay in HCC samples followed by bioinformatic analysis suggested that Metallothionein1G (MT1G) might be one of the key factors in HCC. In this study, we aim to clarify the biological function of MT1G and validate its potential to be utilised as a biomarker in HCC.

Methods We detected the MT1G expression in paired HCC samples and cell lines by both RT-qPCR and Western blot. MSP (Methylation specific PCR) and BGS (Bisulfite genomic sequencing) were performed to evaluate methylation status of MT1G in HCC. TCGA (The Cancer Genome Altas) data analysis was used to validate the results from our samples. The functional significance of MT1G was investigated by overexpression or knockdown in vivo and in vitro. Kaplan-Meier survival analysis was performed using TCGA data to estimate the clinical value of MT1G expression and methylation status in HCC.

Results MT1G was inactivated in 4 of 6 HCC cell lines. The expression of MT1G was downregulated in cancer tissues compared with the adjacent non-tumour tissues $(p<0.001)$. The gene expression of MT1G was closely correlated to the promoter methylation status. The MT1G expression in silenced HCC cell lines could be restored by demethylation agent. Ectopic re-expression of MT1G by stable transfection in SMMC7721 and Hep3B cells inhibited colony formation $(p<0.001)$, suppressed cell motility and invasiveness $(p<0.05)$, accompanying with up-regulation of E-cadherin; and downregulation of PCNA, MMP2, MMP13 and Vimentin. Xenograft tumour assay in nude mice also revealed that MT1G could markedly decrease tumour weights and volumes in vivo $(p<0.001)$. Survival analysis revealed that hypermethylation of MT1G predicts good outcomes of HCC patients.

Conclusions Our results demonstrate that MT1G promoter methylation directly mediates the transcription down-regulation and commonly occurs in HCC. MT1G gene can act as a functional tumour suppressor in liver carcinogenesis by playing an important role in the depression of cell proliferation, migration and invasion.

\section{IDDF2018-ABS-0140 THE CROSSTALK OF MTORC1 AND DNA METHYLATION IN HEPATOCELLULAR CARCINOMA}

${ }^{1}$ Judeng Zeng*, ${ }^{1}$ Meng-ke Chen, ${ }^{2}$ Ning Zhang, ${ }^{2}$ Li-xia Xu, ${ }^{1}$ Yang Yang, ${ }^{1}$ Xiao-yi Xu, ${ }^{1}$ Huiyun Wang, 'Steven Xiao-feng Zheng. 'State Key Laboratory of Oncology in South China, Collaborative Innovation Center for Cancer Medicine, Sun Yat-Sen University Cancer Center, Guangzhou, Guangdong, China; ${ }^{2}$ Department of Gastroenterology, The First Affiliated Hospital, Sun Yat-sen University, Guangzhou, Guangdong, China

\subsection{6/gutjnl-2018-IDDFabstracts.51}

Background Metabolic reprogramming in cancer cells is likely to interact with the epigenetic landmarks, which may modulate tumorigenesis. However, the exact mechanism is still largely unknown. In our previous study, inhibition of activity of mechanistic target of rapamycin (mTORC1), a nutrient sensor that affects cellular metabolism, was able to attenuate the DNA methylation level. In this study, we aim to clarify the correlation between the metabolism and DNA methylation and evaluate their interactive roles in the pathogenesis of hepatocellular carcinoma (HCC).

Methods We treated HCC cell lines with rapamycin to inhibit mTORC1 activity and performed immunofluorescence (IF) and Enzyme-linked immune sorbent assay (ELISA) to detect the levels of 5-methylcytosine (5-mC). Western-blot and IF assays were used to detect the protein levels of DNA methyltransferases (DNMTs) and RT-PCR was performed to test transcription of DNMTs. Cycloheximide was used to suppress the protein translation. Moreover, MG132 and BafA1 were used to inhibit the functions of ubiquitin-proteasome and autophagy-lysosome respectively to evaluate the effect of posttranslational modification for DNMTs degradation. Finally, we selected twenty liver cancer cell lines and combined westernblot and CCK8 assays to observe the association between mTORC1 inhibition-induced DNMTs degradation and cell viabilities.

Results Rapamycin treatment reduced $5 \mathrm{mC}$ levels in HCC cell lines. In parallel, mTORC1 inhibition attenuated the expressions of DNMT1. The subsequent experiments revealed that transcriptional regulation and posttranslational modification is not likely to participate in the process of DNMT1 degradation induced by mTORC1 inhibition. However, cycloheximide half-life assay indicated that mTORC1 might adjust the expression DNMT1 through regulating initial translational efficiency. We found that mTORC1-mediated DNMT1 degradation is closely linked to cell proliferation.

Conclusions In conclusion, we demonstrated that mTORC1 could affect DNA methylation levels in HCC probably through regulating DNMTs expression. Besides, this interactive role seems to be closely linked to HCC cell viability. Investigation of the relationship between mTORC1 signalling and DNA methylation will deepen our insight on the pathogenesis of HCC and develop a novel therapeutic strategy for HCC patients.

\section{IDDF2018-ABS-0165 CIRCULAR RNA CIRC5379-6 PERFORMS FUNCTIONS IN INHIBITING TUMORIGENESIS AND METASTASIS OF HEPATOCELLULAR CARCINOMA VIA THE REGULATION OF PPAR $\alpha$}

${ }^{1}$ Ning Zhang*, ${ }^{1}$ Xin-yue Zhang, ${ }^{2}$ Xiao-xing Li, ${ }^{1}$ Min-hu Chen. ${ }^{1}$ Department of Gastroenterology, The First Affiliated Hospital, Sun Yat-sen University, Guangzhou, Guangdong, China; ${ }^{2}$ State Key Laboratory of Oncology in South China, Collaborative Innovation Center for Cancer Medicine, Sun Yat-Sen University Cancer Center, Guangzhou, Guangdong, China

\subsection{6/gutjnl-2018-IDDFabstracts.52}

Background Circular RNAs (circRNAs) are considered as RNA molecules in loop structure and was reported as a potential novel biomarker for hepatocellular carcinoma (HCC). Peroxisome proliferator-activated receptor alpha (PPAR $\alpha)$ is a suppressor of HCC via direct targeting nuclear factor of kappa light polypeptide gene enhancer in B-cells inhibitor, alpha $(\mathrm{I} \kappa \mathrm{B} \alpha)$ and nuclear factor kappa-light-chain-enhancer of activated $B$ cells $(\mathrm{NF}-\kappa \mathrm{B})$ signalling pathway according to our previous study, whereas their relationship in the modulation of pathogenesis processes in HCC is still unclear. The aim of this study was to determine the circRNAs those perform functions in regulating the gene $\operatorname{PPAR} \alpha$ and thereby regulate the pathogenesis processes in HCC.

Methods The candidate circRNAs were firstly validated in structure via the RNase $\mathrm{R}$ treatment, amplification of specific convergent and divergent primers, and Sanger sequencing. After over-expressing the circRNAs in HCC cell lines and further validation of the cell viability assay, the circRNA correlated to PPAR $\alpha$ was obtained. Functional analysis of the 